\title{
AXIOMATIZATION OF VALUES OF COOPERATIVE GAMES USING A FAIRNESS PROPERTY
}

Abstract. We propose new systems of axioms which characterize four types of values of cooperative games: the Banzhaf value, the Deegan-Packel value, the least square prenucleolus and the least square nucleolus. The common element used in these axiomatizations is a fairness property. It requires that if to a cooperative game we add another game in which two given players are symmetric, then their payoffs change by the same amount. In our analysis we will use an idea applied by R. van den Brink (2001) to obtain an axiomatic characterization of the Shapley value.

Introduction. The article is devoted to axiomatizations of four types of values of cooperative games: the Banzhaf value, the Deegan-Packel value, the least square prenucleolus and the least square nucleolus. In contrast to the existing well known axiomatic theorems concerning these values, we use a fairness property as a common feature of all systems of axioms. This property requires that if to a cooperative game we add another game in which two given players are symmetric, then their payoffs change by the same amount.

Fairness was used by R. van den Brink (2001) to axiomatize the Shapley value. Our article is in a sense a continuation of his research. We will show that by the use of fairness one can obtain a relatively simple collection of conditions describing the four values mentioned above. In the case of linear values we can omit additivity. We employ the dummy player, amalgamation and fairness axioms to characterize the Banzhaf value. In the case of the Deegan-Packel value the system of axioms includes the quasi-efficiency, zeroplayer and fairness properties. Moreover, we will prove that fairness is a

2000 Mathematics Subject Classification: Primary 91A12.

Key words and phrases: cooperative game, value, Banzhaf value, Deegan-Packel value, least square prenucleolus, least square nucleolus. 
good tool to characterize the least square nucleolus, which is not additive and probably for this reason has not been axiomatized yet. This has turned out to be a simple consequence of the axiomatization of the least square prenucleolus, which consists of the efficiency, additivity and fairness axioms and a new inter-game balance axiom. All our new results are presented in Theorems 6-9 at the beginning of Section 2 .

1. Definitions and fundamental facts. In this section we introduce some main notions, assumptions and relevant facts of cooperative game theory.

1.1. Cooperative games and axioms. Let $n$ be a natural number, fixed throughout the paper. An n-person transferable utility cooperative game (briefly: $T U$-game) is defined by the set of players $N=\{1, \ldots, n\}$ (a grand coalition) and by a function $v: 2^{N} \rightarrow \mathbb{R}$ with $v(\emptyset)=0$, called the characteristic function of the game. Therefore, when $N$ is fixed, a TU-game $(N, v)$ can be identified with $v$. A game $v$ is called additive if $v(S \cup K)=v(S)+v(K)$ for any disjoint sets $S, K \subseteq N$, called coalitions. The cardinality of a coalition $S$ will be denoted by $|S|$. The unanimity game $u_{T}, T \subseteq N$, is such that $u_{T}(S)=1$ if $T \subseteq S$ and $u_{T}(S)=0$ otherwise, for any $S \subseteq N$.

A value of the game $v$ is defined to be a function $\varphi(v)=\left(\varphi_{1}(v), \ldots, \varphi_{n}(v)\right)$ which assigns to the game $v$ a vector from $\mathbb{R}^{n}$. An $n$-dimensional vector $x=\left(x_{1}, \ldots, x_{n}\right) \in \mathbb{R}^{n}$ is called a preimputation if

$$
\sum_{i=1}^{n} x_{i}=v(N) .
$$

If, additionally, $x_{i} \geq v(\{i\})$ for all $i \in N$, then $x$ is called an imputation. The sets of all preimputations and imputations of the game $v$ will be denoted by $\mathbf{P}$ and $\mathbf{P I}$, respectively. Let $G_{N}$ be the set of all games $v$.

Now we formulate several fundamental properties used most often in various axiomatic theorems. They are basic for our considerations. The first group of Axioms 1-7 is standard.

Let $\varphi$ be a value on $G_{N}$ and $v \in G_{N}$ be any $n$-person game.

Axiom 1 (Efficiency). A value $\varphi$ is efficient if for any game $v$,

$$
\sum_{i=1}^{n} \varphi_{i}(v)=v(N) .
$$

This means that the vector $\varphi(v)$ is a preimputation.

AxIom 2 (Super-efficiency). A value $\varphi$ is super-efficient if it is efficient and the vector $\varphi(v)$ is an imputation for any game $v$ for which the set PI is nonempty. 
Axiom 3 (Equal treatment). If for two different players $i, j \in N$ and all $S \subseteq N \backslash\{i, j\}$ we have $v(S \cup\{i\})=v(S \cup\{j\}$ ) (in this case players $i$ and $j$ are called symmetric) then $\varphi_{i}(v)=\varphi_{j}(v)$.

Let $v \in G_{N}$ and $\sigma$ be a permutation of the set $N$. We define a new game $\sigma v$ by $\sigma v(\sigma(K))=v(K)$ for any $K \subseteq N$, where $\sigma(K)=\{\sigma(i): i \in K\}$.

Axiom 4 (Symmetry). A value $\varphi$ is symmetric if $\varphi_{\sigma(i)}(\sigma v)=\varphi_{i}(v)$ for any $i \in N$, any game $v$, and any permutation $\sigma$ of the set $N$.

Note that symmetry implies equal treatment (cf. L. S. Shapley (1953)).

Axiom 5 (Additivity). A value $\varphi$ is additive if $\varphi(v+w)=\varphi(v)+\varphi(w)$ for any two $n$-person cooperative games $v, w$, where $(v+w)(S)=v(S)+w(S)$ for any $S \subseteq N$.

Axiom 6 (Dummy player property). If $i \in N$ is a dummy player of the game $v$, i.e. if $v(S \cup\{i\})=v(S)+v(\{i\})$ for any $S \subseteq N \backslash\{i\}$, then $\varphi_{i}(v)=v(\{i\})$.

Axiom 7 (Inessential game property). If $v$ is an additive game then $\varphi_{i}(v)=v(\{i\})$ for any $i \in N$.

The next axiom describes an interesting property which is a common feature of all the values of cooperative games analyzed in the paper. It was introduced by R. van den Brink (2001) to get a new axiomatization of the Shapley value.

Axiom 8 (Fairness). A value $\varphi$ has the fairness property if for any two symmetric players $i$ and $j$ in a game $w$ we have $\varphi_{i}(v+w)-\varphi_{i}(v)=$ $\varphi_{j}(v+w)-\varphi_{j}(v)$ for any game $v$, i.e. the payoffs of both players change by the same amount.

E. Lehrer (1988) introduced the notion of amalgamation of two players and the corresponding new axiom in the description of the Banzhaf value.

Definition 1 (E. Lehrer (1988)). An amalgamation of two different players $i, j$ of an $n$-person game $v$ is a transformation from the game $v$ into the $(n-1)$-person game $v_{(i j)}$ with the set of players $(N \backslash\{i, j\}) \cup\{p\}$, where $p$ denotes a player represented by the coalition $\{i, j\}$. The characteristic function of this game is defined by

$$
v_{(i j)}(K)= \begin{cases}v(K) & \text { if } p \notin K, \\ v((K \backslash\{p\}) \cup\{i, j\}) & \text { if } p \in K,\end{cases}
$$

for any set $K \subseteq(N \backslash\{i, j\}) \cup\{p\}$. 
To formulate the next axiom we need to introduce the set of all games $v$ with grand coalition $S \subseteq N$, that is,

$$
\widetilde{G}_{N}=\bigcup_{S \subseteq N} G_{S} .
$$

Axiom 9 (Amalgamation property). For any two different players $i, j \in$ $N$ and any $v \in \widetilde{G}_{N}, \varphi_{p}\left(v_{(i j)}\right)=\varphi_{i}(v)+\varphi_{j}(v)$.

The next two axioms were used in the axiomatic description of the Deegan-Packel value (J. Deegan and E. W. Packel (1979)).

Axiom 10 (Quasi-efficiency). A value $\varphi$ is quasi-efficient if for any game $v$,

$$
\sum_{i=1}^{n} \varphi_{i}(v)=\sum_{S \subseteq N} v(S) .
$$

Axiom 11 (Zero-player property). If $i \in N$ is a zero-player of a game $v$ then $\varphi_{i}(v)=0$. (A player $i \in N$ is called a zero-player if for any $K \subseteq N$, $v(K)=0$ whenever $i \in K$.)

L. M. Ruiz et al. (1996) proposed a new value, the least square prenucleolus, where the next axiom was used.

For a game $v$ and $i \in N$, we define

$$
g_{i}(v)=\sum_{\substack{K \subseteq N \\ i \in K}} v(K) .
$$

Axiom 12 (Average marginal contribution monotonicity (AMC)). For any $i, j \in N$ and any game $v$, if $g_{i}(v) \geq g_{j}(v)$ then $\varphi_{i}(v) \geq \varphi_{j}(v)$.

The last axiom is new. It is applied in the axiomatization of the least square prenucleolus and the least square nucleolus (values defined by L. M. Ruiz et al. (1996)), proposed in the present paper.

Axiom 13 (Inter-game balance). Let $v, w \in G_{N}$. For any $i, j \in N$ if $\varphi_{i}(v+w)=\varphi_{j}(v+w)$, then $\varphi_{i}(v)-\varphi_{j}(w)=\varphi_{j}(v)-\varphi_{i}(w)$.

This property means that if the values of two players in the sum of two games are equal then the differences between the values of these players in the respective games are opposite (or, in other words, the sums of the values for these players in the two games are equal). It is easy to check that additivity implies inter-game balance. Let $\varphi$ be an additive solution on $G_{N}$. Then for any $v, w \in G_{N}$ and $i, j \in N$ if $\varphi_{i}(v+w)=\varphi_{j}(v+w)$ then $\varphi_{i}(v)+\varphi_{i}(w)=\varphi_{i}(v+w)=\varphi_{j}(v+w)=\varphi_{j}(v)+\varphi_{j}(w)$, which is equivalent to Axiom 13. Therefore the inter-game balance axiom seems weaker than additivity. We show later on that it is satisfied by the least square nucleolus, which is not additive. 
1.2. Basic values and their properties. We first recall formulae for some most important types of values of cooperative games, and next quote some relevant theorems.

Definition 2 (L. S. Shapley (1953)). The Shapley value of player $i \in N$ in a game $v \in G_{N}$ is defined as

$$
\mathrm{Sh}_{i}(v)=\sum_{S \subseteq N} \frac{|S| !(n-|S|-1) !}{n !}(v(S \cup\{i\})-v(S)) .
$$

Definition 3 (J. F. Banzhaf III (1965)). The Banzhaf value of player $i \in N$ in a game $v \in G_{N}$ is defined as

$$
\mathrm{B}_{i}(v)=\frac{1}{2^{n-1}} \sum_{S \subseteq N}(v(S \cup\{i\})-v(S)) .
$$

Definition 4 (J. Deegan and E. W. Packel (1979)). The Deegan-Packel value of player $i \in N$ in a game $v \in G_{N}$ is defined by

$$
\operatorname{DP}_{i}(v)=\sum_{\substack{K \subseteq N \\ i \in K}} \frac{v(K)}{|K|} .
$$

Denote by $S_{1}, \ldots, S_{2 n}$ all the subsets of a set $N$. For a vector $x=$ $\left(x_{1}, \ldots, x_{n}\right) \in \mathbb{R}^{n}$ and a coalition $S$ we define

$$
x(S)=\sum_{i \in S} x_{i} .
$$

A vector $r(v, x) \in \mathbb{R}^{2^{n}}$ with coordinates of the form

$$
r_{i}(v, x)=v\left(S_{i}\right)-x\left(S_{i}\right) \quad \text { for } i=1, \ldots, 2^{n}
$$

is called the excess vector.

Definition 5 (L. M. Ruiz et al. (1996)). The least square prenucleolus (briefly: LS-prenucleolus) of a game $v \in G_{N}$ is a preimputation $x$ of this game which satisfies

$$
\sum_{i=1}^{2^{n}}\left(r_{i}(v, x)-\overline{r(v, x)}\right)^{2}=\min _{y \in \mathbf{P}} \sum_{i=1}^{2^{n}}\left(r_{i}(v, y)-\overline{r(v, y)}\right)^{2},
$$

where $\overline{r(v, x)}$ denotes the arithmetic mean of all coordinates of $r(v, x)$.

Definition 6 (L. M. Ruiz et al. (1996)). The least square nucleolus (briefly: $L S$-nucleolus) of a game $v \in G_{N}$ is an imputation $x$ of this game which satisfies

$$
\sum_{i=1}^{2^{n}}\left(r_{i}(v, x)-\overline{r(v, x)}\right)^{2}=\min _{y \in \mathbf{P I}} \sum_{i=1}^{2^{n}}\left(r_{i}(v, y)-\overline{r(v, y)}\right)^{2} .
$$


Now we will quote several theorems characterizing basic values defined above. During the last fifty years many axiomatizations based on various sets of properties have been formulated and proved. We have chosen only those results which will be useful in our research.

The well known axiomatization of the Shapley value (L. S. Shapley (1953)) states that $\operatorname{Sh}(v)$ is the unique value satisfying the efficiency, dummy player, equal treatment and additivity axioms. R. van den Brink (2001) proved that equal treatment and additivity can be replaced by the fairness axiom.

Theorem 1. A value $\varphi$ on $G_{N}$ satisfies the efficiency, dummy player and fairness axioms if and only if $\varphi(v)=\operatorname{Sh}(v)$ for all $v \in G_{N}$.

REMARK. The first axiomatization of the Shapley value without the additivity axiom was given by H. P. Young (1985). Moreover, R. van den Brink (2001) noted some connections between fairness and other axioms. We state them together with a third simple property in the following theorem.

TheOREM 2. Let $\varphi$ be a value on $G_{N}$.

(a) If $\varphi$ satisfies the equal treatment and additivity axioms, then it also satisfies the fairness axiom.

(b) If $\varphi$ satisfies the dummy player and fairness axioms, then it also satisfies the equal treatment axiom.

(c) If $\varphi$ satisfies the zero-player and fairness axioms, then it also satisfies the equal treatment axiom.

In general, the Banzhaf value $\mathrm{B}(v)$ is not efficient. On account of this difficulty, the first axiomatization of this value was given relatively late. E. Lehrer based his important result on the amalgamation axiom.

Theorem 3 (E. Lehrer (1988)). The Banzhaf value $\varphi(v)=\mathrm{B}(v)$ for all $v \in G_{N}$ is the unique value on $\widetilde{G}_{N}$ which satisfies the dummy player, equal treatment, amalgamation and additivity axioms.

Theorem 4 (J. Deegan and E. W. Packel (1979)). The value $\varphi(v)=$ $\operatorname{DP}(v)$ for all $v \in G_{N}$ is the unique value on $G_{N}$ which satisfies the symmetry, quasi-efficiency, zero-player and additivity axioms.

L. M. Ruiz et al. (1996) proved that for any $v \in G_{N}$ and any player $i \in N$ the LS-prenucleolus $\mathrm{L}(v)$ can be expressed by

$$
\mathrm{L}_{i}(v)=\frac{v(N)}{n}+\frac{1}{n 2^{n-2}}\left(n g_{i}(v)-\sum_{d \in N} g_{d}(v)\right) .
$$

It is worth noting that the LS-prenucleolus is the additive normalization of the Banzhaf value, i.e., for $i \in N$, 


$$
\mathrm{L}_{i}(v)=\mathrm{B}_{i}(v)+\left(v(N)-\sum_{d=1}^{n} \mathrm{~B}_{d}(v)\right) / n .
$$

In the article by L. M. Ruiz et al. (1996) the following was proved.

THEOREM 5. The LS-prenucleolus is the unique value on $G_{N}$ which satisfies the efficiency, average marginal contribution monotonicity, inessential game and additivity axioms.

The fact that the LS-nucleolus is not additive is the main reason for the lack of its effective axiomatization. In this article we will try to provide some consistent set of axioms to characterize this value.

The LS-nucleolus can be calculated using the following algorithm.

Algorithm 1 (L. M. Ruiz et al. (1996)). Construct a sequence of pairs $\left(\mathbf{x}_{i}, T_{i}\right), i=1,2, \ldots$, where $\mathbf{x}_{i}=\left(x_{i 1}, \ldots, x_{i n}\right)$ is a preimputation and $T_{i}$ is a subset of $N$ inductively defined by

(a) $\mathbf{x}_{1}:=L(v)$ and $T_{i}:=\left\{j \in N: x_{i j}<v(\{j\})\right\}$,

(b) $\mathbf{x}_{i+1}$ is the solution of the problem

$$
\begin{aligned}
& \min \sum_{S \subseteq N}(v(S)-y(S))^{2} \\
& \quad \text { such that } \sum_{j \in N} y_{j}=v(N) \text { and } y_{j}=v(\{j\}) \forall j \in M_{i}=\bigcup_{k=1}^{i} T_{k} .
\end{aligned}
$$

The sequence terminates when $T_{i}=\emptyset$. This procedure ends after at most $n-1$ steps and the final vector $\mathbf{x}_{i}$ is the LS-nucleolus. One can easily check that the solution $\mathbf{x}_{i+1}=\left(y_{1}, \ldots, y_{n}\right)$ of the problem in (b) for $j \notin M_{i}$ is of the form

$$
\mathbf{x}_{i+1, j}=\frac{v(N)-\mathbf{x}_{i}\left(M_{i}\right)}{n-\left|M_{i}\right|}+\frac{1}{\left(n-\left|M_{i}\right|\right) 2^{n-2}}\left(\left(n-\left|M_{i}\right|\right) g_{i}(v)-\sum_{k \notin M_{i}} g_{k}(v)\right)
$$

for every $i=2,3, \ldots$.

2. Main results. As mentioned earlier, the fairness axiom is a useful property describing a feature of "just" cooperative games and used to construct a specific axiomatization of the Shapley value (cf. Theorem 1). It turns out, however, that this axiom is also very helpful in an axiomatic description of other values: the Banzhaf value, the Deegan-Packel value, the LS-prenucleolus and the LS-nucleolus. As those values are completely different from one another, this can be rather surprising. Below we formulate Theorems 6-9, describing the four values axiomatically with the use of the fairness axiom. 
The first theorem shows that in the classical axiomatic characterization of the Banzhaf value (cf. Theorem 3) the equal treatment and additivity properties can be replaced by the fairness axiom.

Theorem 6. A value $\varphi$ on $\widetilde{G}_{N}$ coincides with the Banzhaf value if and only if it satisfies the amalgamation, dummy player and fairness axioms.

In our second theorem a new axiomatization of the Deegan-Packel value consists of two axioms used in the classical approach (cf. Theorem 3) and the fairness property instead of symmetry and additivity.

TheOREM 7. The Deegan-Packel value is the unique value on $G_{N}$ which satisfies the quasi-efficiency, zero-player and fairness axioms.

The third theorem gives an axiomatization of the LS-prenucleolus. It is based on the fairness axiom and on the inter-game balance property (cf. Axiom 13).

TheOREM 8. A value $\varphi$ on $G_{N}$ satisfies the efficiency, inter-game balance, average marginal contribution monotonicity, fairness and inessential game axioms if and only if $\varphi$ is the LS-prenucleolus.

The last theorem gives an axiomatic description of the LS-nucleolus; it is a simple consequence of Theorem 8 . This is the first effective axiomatization of this value.

TheOREM 9. A value $\varphi$ on $G_{N}$ satisfies the super-efficiency, inter-game balance, average marginal contribution monotonicity, fairness and inessential game axioms if and only if $\varphi$ is the LS-nucleolus.

The proofs of Theorems 6-9 are given in Subsections 2.1-2.4.

2.1. Proof of Theorem 6 . We begin with the following lemma.

LEMma 1. Assume that a value $\varphi$ on $\widetilde{G}_{N}$ satisfies the amalgamation, dummy player and fairness axioms. Then for any nonempty coalition $T \subseteq N$, unanimity game $u_{T}$ and any constant $c$,

$$
\varphi_{i}\left(c \cdot u_{T}\right)= \begin{cases}c / 2^{|T|-1} & \text { if } i \in T, \\ 0 & \text { if } i \notin T .\end{cases}
$$

Proof. The proof is similar to the corresponding proofs for other axiomatizations (A. S. Nowak (1997), for example). It will be carried out by induction on $t, 1 \leq t \leq n$. First notice that $\varphi_{i}\left(c \cdot u_{T}\right)$ of the form $(2)$ coincides with the Banzhaf value $\mathrm{B}_{i}\left(c_{i} \cdot u_{T}\right)$. This follows easily from Definition 3 .

Formula (2) holds for $|T|=1$, because then each player in the game $u_{T}$ is dummy. Suppose that it is true for some $n$ and for any coalition $T$ with $|T|=t<n$. Let $\omega_{\mathcal{T}}$ be an $n$-person unanimity game with $|\mathcal{T}|=$ $t+1$. Amalgamate some two different players $i, j \in \mathcal{T}$. Then according to 
Definition $1, \omega_{\mathcal{T}(i j)}=\omega_{\mathcal{T} *}$ is the $n$-person unanimity game of the coalition $\mathcal{T}^{*}=(\mathcal{T} \backslash\{i, j\}) \cup\{p\}$ with $\left|\mathcal{T}^{*}\right|=t$.

By the induction hypothesis $\varphi_{p}\left(c \cdot \omega_{\mathcal{T}^{*}}\right)=c / 2^{\left|\mathcal{T}^{*}\right|-1}=c / 2^{t-1}$ and according to the amalgamation property $\varphi_{i}\left(c \cdot \omega_{\mathcal{T}}\right)+\varphi_{j}\left(c \cdot \omega_{\mathcal{T}}\right)=c / 2^{t-1}$.

The fairness and dummy player axioms imply equal treatment (by Theorem $2(\mathrm{~b})$ ). Hence, since players $i$ and $j$ are symmetric in the game $c \cdot \omega_{\mathcal{T}}$, we have $\varphi_{i}\left(c \cdot \omega_{\mathcal{T}}\right)=\varphi_{j}\left(c \cdot \omega_{\mathcal{T}}\right)$, and consequently, $\varphi_{i}\left(c \cdot \omega_{\mathcal{T}}\right)=c / 2^{t}=c / 2^{|\mathcal{T}|-1}$ for $i \in \mathcal{T}$.

On the other hand, the dummy player axiom implies that $\varphi_{i}\left(c \cdot \omega_{\mathcal{T}}\right)=0$ if $i \notin \mathcal{T}$. Therefore, by the induction principle, formula (2) holds. Thus $\varphi\left(c \cdot u_{T}\right)=\mathrm{B}\left(c \cdot u_{T}\right)$ for any unanimity game $u_{T}$ and any real $c$, ending the proof of the lemma.

In the proof of the theorem, we will use the game graph method proposed by R. van den Brink (2001) for the Shapley value. For completeness we repeat some arguments from that paper.

$(\Rightarrow)$ The Banzhaf value satisfies the amalgamation and dummy players axioms (cf. Theorem 3). Because it is additive and has the equal treatment property, according to Theorem 2(a), it also satisfies fairness.

$(\Leftarrow)$ Fix a value $\varphi$ on $\widetilde{G}_{N}$ and assume that it has all the properties mentioned in the theorem.

We will prove that $\varphi=\mathrm{B}$. It is clear that $\varphi=\mathrm{B}$ for all one-player games (in this case $v=c \cdot u_{\{i\}}$ for some real number $c$ ). Suppose that $\varphi=\mathrm{B}$ for all $m$-person games where $m \leq n-1$. Let then $v$ be an $n$-person game. It can be uniquely represented as a linear combination of unanimity games (cf. L. S. Shapley (1953)):

$$
v=\sum_{S \subseteq N} \eta_{S} u_{S}
$$

where $\eta_{S}$ are constants. Let $\delta(v)$ be the number of nonzero coefficients $\eta_{S}$ in (3). We will use induction on $\delta(v)$.

If $\delta(v)=0$, then $v$ is the null game, i.e. $v(S)=0$ for all $S \subseteq N$. The dummy player property implies that $\varphi_{i}(v)=0=\mathrm{B}_{i}(v)$ for any $i \in N$. If $\delta(v)=1$ then $v=\eta_{S} u_{S}$ for some $S \subseteq N$ with $\eta_{S} \neq 0$. Then we have $\varphi_{i}(v)=\mathrm{B}_{i}\left(\eta_{S} u_{S}\right)$ for any $i \in N$.

Now assume that $\varphi(z)$ is uniquely determined and coincides with $\mathrm{B}(z)$ for all games $z \in G_{N}$ with $\delta(z) \leq k(k \geq 1)$, and let $v$ be any $n$-person game with $\delta(v)=k+1$. Then $\delta(v) \geq 2$ and $n \geq 2$. Put $\Delta(v)=\left\{S \subseteq N: \eta_{S} \neq 0\right\}$. We introduce the following auxiliary definition.

Definition 7. Define the graph $\left(N, \Re_{v}\right)$ as follows: $\{i, j\} \in \Re_{v}$ if and only if $i, j \in N, i \neq j$ and there exists a set $S \in \Delta(v)$ with $\{i, j\} \subseteq S$ or $\{i, j\} \cap S=\emptyset$. A coalition $K \subseteq N$ is said to be connected in $\Re_{v}$ if 
either $\operatorname{card}(K)=1$ or for any $i, j \in K, i \neq j$, there is a sequence of players $p_{1}, \ldots, p_{q}$ such that $p_{1}=i, p_{q}=j$ and $\left\{p_{r}, p_{r+1}\right\} \in \Re_{v}$ for all $r \in\{1, \ldots, q-1\}$. A connected coalition $K$ is a component in $\Re_{v}$ if $\{i, j\} \notin \Re_{v}$ whenever $i \in K$ and $j \in N \backslash K$.

The rest of the proof will be divided into two cases.

CASE 1: $N$ is a component in $\Re_{v}$, i.e. $\left(N, \Re_{v}\right)$ is a connected graph. Let $\zeta(v)=\left\{i \in N: \eta_{S} \neq 0\right.$ for some $\left.S \ni i\right\}$. Fix any $j \in \zeta(v)$ and let $S_{0}=\{j\}$. For $b \in N$ we define recursively the sets

(4) $S_{b}=\left\{i \in N \backslash \bigcup_{\mu=0}^{b-1} S_{\mu}\right.$ : there exists an $h \in S_{b-1}$ such that $\left.\{i, h\} \in \Re_{v}\right\}$. Clearly, $S_{r} \cap S_{t}=\emptyset$ for $r \neq t$. Suppose that $N \backslash \bigcup_{\mu=0}^{b-1} S_{\mu} \neq \emptyset$ and $S_{b}=\emptyset$. Then for any $i \in N \backslash \bigcup_{\mu=0}^{b-1} S_{\mu}$ and $h \in \bigcup_{\mu=0}^{b-1} S_{\mu}$ we have $\{i, h\} \notin \Re_{v}$, which is impossible, because $N$ is a component in $\Re_{v}$. Thus $N \backslash \bigcup_{\mu=0}^{b-1} S_{\mu}=\emptyset$.

Therefore the finiteness of $N$ implies that there exists an $m \in N$ such that $S_{0}, S_{1}, \ldots, S_{m}$ is a partition of $N$ consisting of nonempty sets.

Let $\varphi_{j}(v)=a$ for some $a \in \mathbb{R}$ and let $a_{j}=0$. Therefore we can put

$$
\varphi_{i}(v)=a+a_{i}, \quad i \in N,
$$

for some $a_{i}$. Clearly, the set $\left\{a_{i}: i \in S_{0}\right\}$ is uniquely determined, since $S_{0}=\{j\}$ and $a_{j}=0$. Suppose now that all $a_{h}$ for $h \in S_{t-1}$, for some $t \geq 1$, have been uniquely determined.

For any fixed $i \in S_{t}$, there exist $h \in S_{t-1}$ and $S \in \Delta(v)$ such that $\{i, h\} \subseteq S$ or $\{i, h\} \cap S=\emptyset$. Then players $i, h$ are symmetric in the game $\eta_{S} u_{S}$ and from the fairness property we have $\varphi_{i}(v)-\varphi_{i}\left(v-\eta_{S} u_{S}\right)=\varphi_{h}(v)-$ $\varphi_{h}\left(v-\eta_{S} u_{S}\right)$. Hence,

$$
\begin{aligned}
a+a_{i} & =\varphi_{i}(v)=\varphi_{h}(v)-\varphi_{h}\left(v-\eta_{S} u_{S}\right)+\varphi_{i}\left(v-\eta_{S} u_{S}\right) \\
& =a+a_{h}-\varphi_{h}\left(v-\eta_{S} u_{S}\right)+\varphi_{i}\left(v-\eta_{S} u_{S}\right)
\end{aligned}
$$

and therefore $a_{i}=a_{h}-\varphi_{h}\left(v-\eta_{S} u_{S}\right)+\varphi_{i}\left(v-\eta_{S} u_{S}\right)$. However, by our main induction hypothesis (i.e. $\delta\left(v-\eta_{S} u_{S}\right)=k$ ) we have $\varphi_{h}\left(v-\eta_{S} u_{S}\right)=$ $\mathrm{B}_{h}\left(v-\eta_{S} u_{S}\right)$ and $\varphi_{i}\left(v-\eta_{S} u_{S}\right)=\mathrm{B}_{i}\left(v-\eta_{S} u_{S}\right)$. It follows, therefore, that $a_{i}$ is uniquely determined.

Amalgamate now players $i$ and $h$. Then the amalgamation axiom and induction hypothesis imply

$$
2 a+a_{i}+a_{h}=\varphi_{i}(v)+\varphi_{h}(v)=\varphi_{p}\left(v_{(i h)}\right)=\mathrm{B}_{p}\left(v_{(i h)}\right) .
$$

Consequently, also $a=\left(\mathrm{B}_{p}\left(v_{(i h)}\right)-a_{i}-a_{h}\right) / 2$ is uniquely determined. Summarizing, all values $\varphi_{i}(v), i=1, \ldots, n$, are uniquely determined. Thus $\varphi_{i}(v)=\mathrm{B}_{i}(v)$ for any $i=1, \ldots, n$, because $\mathrm{B}(v)$ satisfies the three axioms considered in Theorem 6 (cf. Theorems 3 and 2(a)). 
CASE 2: $N$ is not a component in $\Re_{v}$. Then $\left(N, \Re_{v}\right)$ is not a connected graph. This implies that $\Re_{v}$ has at least two components. Moreover, any distinct components are disjoint. We will prove that $\Re_{v}$ has exactly two components. Suppose $B_{1}, B_{2}$ and $B_{3}$ are three distinct components in $\Re_{v}$. Because $\delta(v) \geq 2$ we have $\Delta(v) \neq \emptyset$. Without loss of generality we can assume that there exists $S \in \Delta(v)$ such that $S \subseteq B_{3}$. If $i \in B_{1}$ and $j \in B_{2}$ then $\{i, j\} \cap S=\emptyset$ since different components are disjoint. But then $\{i, j\} \in \Re_{v}$, yielding a contradiction with $B_{1}, B_{2}$ being distinct components in $\Re_{v}$.

Let $S$ and $T$ be two components in $\Re_{v}$. Then $S \cap T=\emptyset$. If $S \cup T \neq N$, then for $i \in S, j \in T$ and $h \in N \backslash(S \cup T)$ we have $\{\{i, h\},\{j, h\}\} \subseteq \Re_{v}$, which is a contradiction with $T$ and $S$ being components in $\Re_{v}$. Because $\delta(v) \geq 2$, $v$ is the sum of two multiplied unanimity games, i.e. $v=\eta_{S} u_{S}+\eta_{T} u_{T}$, where $\eta_{S} \neq 0$ and $\eta_{T} \neq 0$.

We will analyze two subcases according to the cardinality of the set $N$.

First let $n \geq 3$. Without loss of generality we can assume that $|S| \geq 2$. Take $i \in S$ and $j \in T$ and define the game $\xi \in G_{N}$ as

$$
\xi=v+\eta_{S} u_{(S \backslash\{i\}) \cup\{j\}}=\eta_{S} u_{S}+\eta_{S} u_{(S \backslash\{i\}) \cup\{j\}}+\eta_{T} u_{T} .
$$

Now we determine $\varphi(\xi)$. Let $\varphi_{j}(\xi)=a$. By fairness $\left({ }^{1}\right)$ we have $\varphi_{i}(\xi)-$ $\varphi_{i}\left(\eta_{T} u_{T}\right)=\varphi_{j}(\xi)-\varphi_{j}\left(\eta_{T} u_{T}\right)$. We know by $(2)$ that $\varphi_{i}\left(\eta_{T} u_{T}\right)=0$ and $\varphi_{j}\left(\eta_{T} u_{T}\right)=\eta_{T} /\left(2^{|T|-1}\right)$. This way, $\varphi_{i}(\xi)=\varphi_{j}(\xi)-\varphi_{j}\left(\eta_{T} u_{T}\right)+\varphi_{i}\left(\eta_{T} u_{T}\right)$ $=a-\eta_{T} /\left(2^{|T|-1}\right)$. The dummy player axiom implies that

$$
\varphi_{h}\left(\eta_{S} u_{S}+\eta_{S} u_{(S \backslash\{i\}) \cup\{j\}}\right)=0 \quad \text { for } h \in T \backslash\{j\} .
$$

Fairness $\left({ }^{2}\right)$ and the fact that $\delta\left(\eta_{S} u_{(S \backslash\{i\}) \cup\{j\}}\right)=1$ imply that for $h \in$ $T \backslash\{j\}$,

$$
\begin{aligned}
\varphi_{j}\left(\eta_{S} u_{S}+\eta_{S} u_{(S \backslash\{i\}) \cup\{j\}}\right)= & \varphi_{h}\left(\eta_{S} u_{S}+\eta_{S} u_{(S \backslash\{i\}) \cup\{j\}}\right)-\varphi_{h}\left(\eta_{S} u_{(S \backslash\{i\}) \cup\{j\}}\right) \\
& +\varphi_{j}\left(\eta_{S} u_{(S \backslash\{i\}) \cup\{j\}}\right)=\eta_{S} / 2^{|S|-1} .
\end{aligned}
$$

Thus, for any $h \in T \backslash\{j\}$ by fairness $\left(^{3}\right)$ we have

$$
\begin{aligned}
\varphi_{h}(\xi) & =\varphi_{j}(\xi)-\varphi_{j}\left(\eta_{S} u_{S}+\eta_{S} u_{(S \backslash\{i\}) \cup\{j\}}\right)+\varphi_{h}\left(\eta_{S} u_{S}+\eta_{S} u_{(S \backslash\{i\}) \cup\{j\}}\right) \\
& =a-\eta_{S} / 2^{|S|-1} .
\end{aligned}
$$

For any $h \in S \backslash\{i\}$ by fairness $\left({ }^{4}\right)$ we have $\varphi_{h}(\xi)-\varphi_{h}\left(\eta_{S} u_{(S \backslash\{i\}) \cup\{j\}}\right)$ $=\varphi_{i}(\xi)-\varphi_{i}\left(\eta_{S} u_{(S \backslash\{i\}) \cup\{j\}}\right)$. We know that $\varphi_{i}\left(\eta_{S} u_{(S \backslash\{i\}) \cup\{j\}}\right)=0$ and $\varphi_{h}\left(\eta_{S} u_{(S \backslash\{i\}) \cup\{j\}}\right)=\eta_{S} / 2^{|S|-1}$ for any $h \in S \backslash\{i\}$. Thus

$\left.{ }^{1}\right)$ Players $i$ and $j$ are symmetric in the game $\eta_{S} u_{S}+\eta_{S} u_{(S \backslash\{i\}) \cup\{j\}}$.

$\left({ }^{2}\right)$ Players $h$ and $j$ are symmetric in the game $\eta_{S} u_{S}$.

$\left({ }^{3}\right)$ Players $h$ and $j$ are symmetric in the game $\eta_{T} u_{T}$.

$\left({ }^{4}\right)$ Players $i$ and $h$ are symmetric in the game $v$. 


$$
\begin{aligned}
\varphi_{h}(\xi) & =\varphi_{i}(\xi)-\varphi_{i}\left(\eta_{S} u_{(S \backslash\{i\}) \cup\{j\}}\right)+\varphi_{h}\left(\eta_{S} u_{(S \backslash\{i\}) \cup\{j\}}\right) \\
& =a-\eta_{T} / 2^{|T|-1}+\eta_{S} / 2^{|S|-1}
\end{aligned}
$$

for any $h \in S \backslash\{i\}$.

We can now recapitulate our considerations as follows:

$$
\varphi_{h}(\xi)= \begin{cases}a & \text { if } h=j, \\ a-\eta_{T} / 2^{|T|-1} & \text { if } h=i, \\ a-\eta_{S} / 2^{|S|-1} & \text { if } h \in T \backslash\{j\}, \\ a-\eta_{T} / 2^{|T|-1}+\eta_{S} / 2^{|S|-1} & \text { if } h \in S \backslash\{i\} .\end{cases}
$$

Let $i \neq \theta \in S$. Amalgamate players $h$ and $\theta$. Then $\theta \in S \backslash\{i\}$ and by the amalgamation axiom as well as the induction hypothesis we have

$$
\begin{aligned}
\mathrm{B}_{p}\left(\xi_{(h \theta)}\right)= & \varphi_{p}\left(\xi_{(h \theta)}\right)=\varphi_{h}(\xi)+\varphi_{\theta}(\xi) \\
= & \begin{cases}2 a-\eta_{T} / 2^{|T|-1}+\eta_{S} / 2^{|S|-1} & \text { if } h=j, \\
2 a-\eta_{T} / 2^{|T|-2}+\eta_{S} / 2^{|S|-1} & \text { if } h=i, \\
2 a-\eta_{T} / 2^{|T|-1} & \text { if } h \in T \backslash\{j\}, \\
2 a-\eta_{T} / 2^{|T|-2}+\eta_{S} / 2^{|S|-2} & \text { if } h \in S \backslash\{i\} .\end{cases}
\end{aligned}
$$

Calculating $a$ in each of these cases we obtain

$$
\varphi_{h}(\xi)= \begin{cases}\mathrm{B}_{p}\left(\xi_{p}\right) / 2+\eta_{T} / 2^{|T|}-\eta_{S} / 2^{|S|} & \text { if } h=j, \\ \mathrm{~B}_{p}\left(\xi_{p}\right) / 2+\eta_{T} / 2^{|T|-1}-\eta_{S} / 2^{|S|} & \text { if } h=i, \\ \mathrm{~B}_{p}\left(\xi_{p}\right) / 2+\eta_{T} / 2^{|T|} & \text { if } h \in T \backslash\{j\}, \\ \mathrm{B}_{p}\left(\xi_{p}\right) / 2+\eta_{T} / 2^{|T|-1}-\eta_{S} / 2^{|S|-1} & \text { if } h \in S \backslash\{i\} .\end{cases}
$$

Now we must determine the value $\varphi_{i}(v), i \in N$. Let $\varphi_{j}(v)=b$. Fairness and the dummy player property imply equal treatment (by Theorem 2(b)) and therefore $\varphi_{h}(v)=b$ for all $h \in T$. For any $h \in S \backslash\{i\}$ by fairness (players $h$ and $j$ are symmetric in the game $\left.\eta_{S} u_{(S \backslash\{i\}) \cup\{j\}}\right)$ we have $\varphi_{h}(v)-\varphi_{h}(\xi)=$ $\varphi_{j}(v)-\varphi_{j}(\xi)$, and by $(6)$

$$
\varphi_{h}(v)=\varphi_{j}(v)-\varphi_{j}(\xi)+\varphi_{h}(\xi)=b+\left(\eta_{T} / 2^{|T|-1}-\eta_{S} / 2^{|S|-1}\right) / 2 .
$$

From Theorem 2(b) we can also conclude that $\varphi_{h}(v)=\varphi_{d}(v)$ for all $d \in S$, since any two players in $S$ are symmetric in the game $v$. This way

$$
\varphi_{i}(v)= \begin{cases}b+\eta_{T} / 2^{|T|}-\eta_{S} / 2^{|S|} & \text { if } i \in S, \\ b & \text { if } i \in T .\end{cases}
$$

Now, amalgamate two players $i \in S$ and $j \in T$. Then according to the amalgamation axiom and induction hypothesis we obtain $\mathrm{B}_{p}\left(v_{(i j)}\right)=$ $\varphi_{i}(v)+\varphi_{j}(v)=2 b+\eta_{T} / 2^{|T|}-\eta_{S} / 2^{|S|}$, whence $b=\mathrm{B}_{p}\left(v_{(i j)}\right) / 2-\eta_{T} / 2^{|T|+1}+$ $\eta_{S} / 2^{|S|+1}$. Therefore, it follows by $(7)$ that $\varphi_{i}(v)$ is uniquely determined for any $i \in N$, when $n \geq 3$. 
Suppose now that $n=2$. Let $N=\{1,2\}$ and repeat the considerations of the previous subcase with obvious modifications $(|S|=|T|=1)$.

Thus $\varphi(v)$ is uniquely determined and because it satisfies all axioms mentioned in the theorem, we also have $\varphi(v)=\mathrm{B}(v)$, as in Case 1 .

2.2. Proof of Theorem 7 . In the proof we again apply the graph method using a new system of basic games in $\mathbb{R}^{2^{n}}$ instead of unanimity games. The idea is similar to the standard R. van den Brink method.

$(\Rightarrow)$ The Deegan-Packel value (DP) has the quasi-efficiency and zeroplayer properties (cf. Theorem 4). Because it also satisfies equal treatment (an easily verifiable fact) and additivity, it satisfies fairness (by Theorem $2(\mathrm{a}))$.

$(\Leftarrow)$ Fix a value $\varphi$ on $G_{N}$ and assume that it satisfies all the three properties appearing in Theorem 7 . For $S \subseteq N$, let $w_{S}$ be a basic game, i.e.

$$
w_{s}(K)=\left\{\begin{array}{ll}
1 & \text { if } K=S \\
0 & \text { if } K \neq S
\end{array} \quad \text { for any } K \subseteq N .\right.
$$

Then according to quasi-efficiency, zero-player and equal treatment (a consequence of Theorem $2(\mathrm{~b})$ ), we have

$$
\varphi_{i}\left(c \cdot w_{s}\right)=\left\{\begin{array}{ll}
c /|S| & \text { if } i \in S \\
0 & \text { if } i \notin S
\end{array} \quad \text { for any constant } c .\right.
$$

We will prove that $\varphi=$ DP. It is clear that $\varphi=$ DP for all one-player games. Suppose that $\varphi=\mathrm{DP}$ for all $m$-person games where $m \leq n-1$, and let $v$ be any $n$-person game. It is known (cf. J. Deegan and E. W. Packel (1979)) that $v$ can be uniquely represented as a linear combination

$$
v=\sum_{S \subseteq N} c_{S} w_{S}
$$

where $c_{S}$ are constants. Let $d(v)$ be the number of nonzero coefficients $c_{S}$ in (10). We will use induction on $d(v)$.

If $d(v)=0$, then by the zero-player axiom, $\varphi_{i}(v)=0=\mathrm{DP}_{i}(v)$ for any $i \in N$. If $d(v)=1$ then $v=c_{S} \cdot w_{S}$ for some $S \subseteq N$. By earlier conclusions we have $\varphi_{i}(v)=c_{S} /|S|=\mathrm{DP}_{i}\left(c_{S} \cdot w_{S}\right)$ for any $i \in S$ and $\varphi_{i}(v)=0=\mathrm{DP}_{i}\left(c_{S} \cdot w_{S}\right)$ for any $i \in N \backslash S$.

The game graph in Subsection 2.1 was constructed for the structure of unanimity games. It is easy to obtain a similar construction for the representation consisting of the basic games $\left\{w_{S}\right\}$. Assume that $\varphi(z)$ coincides with $\operatorname{DP}(z)$ for all games $z \in G_{N}$ with $d(z) \leq k(k \geq 1)$ and let $d(v)=k+1$. Then $d(v) \geq 2$ and $n \geq 2$. Let $D(v)=\left\{S \subseteq N: c_{S} \neq 0\right\}$. Define the graph $\left(N, \Gamma_{v}\right)$ as in Definition 7 with $D(v)$ instead of $\Delta(v)$. We consider two cases.

CASE 1: $N$ is a component in $\Gamma_{v}$. Let $\vartheta(v)=\left\{i \in N: c_{S} \neq 0\right.$ for some $S \ni i\}$. Fix any $j \in \vartheta(v)$ and let $S_{0}=\{j\}$. For $b \in N$, we define recursively 
the sets $S_{b}$ as in (4), where $\Re_{v}$ is replaced by $\Gamma_{v}$. As in Subsection 2.1 we can prove that there is $m \in N$ such that $S_{0}, S_{1}, \ldots, S_{m}$ is a partition of $N$ consisting of nonempty sets. We can also repeat the further considerations for $i \in S_{t}$ and some $h \in S_{t-1}$ to get $a_{i}=a_{h}-\mathrm{DP}_{h}\left(v-c_{S} \cdot w_{S}\right)+\mathrm{DP}_{i}\left(v-c_{S} \cdot w_{S}\right)$, with some constant $a_{h}$ determined inductively. Hence, all $a_{i}$ with $i \in S_{t}$ are uniquely determined, implying the same for all $a_{i}, i \in N$.

Quasi-efficiency implies that

$$
\sum_{i=1}^{n} \varphi_{i}(v)=n \cdot a+\sum_{i=1}^{n} a_{i}=\sum_{S \subseteq N} v(S) .
$$

Therefore the constant $a$ is also uniquely determined. By the equalities $\varphi_{i}(v)=a+a_{i}$ for $i \in N$, we conclude that all values $\varphi_{i}(v), i=1, \ldots, n$, are uniquely determined. Thus $\varphi_{i}(v)=\mathrm{DP}_{i}(v)$ for any $i=1, \ldots, n$, because DP satisfies the three axioms considered in Theorem 7 (see part $(\Rightarrow)$ ).

CASE 2: $N$ is not a component in $\Gamma_{v}$. In exactly the same way as in Case 2 in the previous subsection, we conclude that there are coalitions $S$ and $T$ with $S \cap T=\emptyset$ and $S \cup T=N$ such that $v=c_{S} w_{S}+c_{T} w_{T}$ with $c_{S} \neq 0$ and $c_{T} \neq 0$. We consider two subcases.

If $n \geq 3$, then without loss of generality we can assume that $|S| \geq 2$. Take an $i \in S$ and $j \in T$ and define the game $\chi \in G_{N}$ as

$$
\chi=v+c_{S} w_{(S \backslash\{i\}) \cup\{j\}}=c_{S} w_{S}+c_{S} w_{(S \backslash\{i\}) \cup\{j\}}+c_{T} w_{T} .
$$

Now, we can repeat the reasoning of the analogous subcase leading to formula (5), replacing $\xi, u,(2)$ and the dummy player axiom by $\chi, w,(10)$ and the zero-player axiom, respectively. Then the result, instead of (5), will be

$$
\varphi_{h}(\chi)= \begin{cases}a & \text { if } h=j, \\ a-c_{T} /|T| & \text { if } h=i, \\ a-c_{S} /|S| & \text { if } h \in T \backslash\{j\}, \\ a-c_{T} /|T|+c_{S} /|S| & \text { if } h \in S \backslash\{i\} .\end{cases}
$$

By quasi-efficiency we have

$$
2 c_{S}+c_{T}=\sum_{S \subseteq N} \chi(S)=\sum_{i=1}^{n} \varphi_{i}(v)=n \cdot a-\frac{|S|}{|T|} c_{T}+\frac{|S|-|T|}{|S|} c_{S} .
$$

Hence the constant $a$, and thereby value $\varphi_{h}(\chi)$ for $h \in N$, is uniquely determined.

Now we must determine $\varphi_{i}(v), i \in N$. Let $\varphi_{j}(v)=b$. Fairness and the zero-player property imply equal treatment (by Theorem 2(c)) and therefore $\varphi_{h}(v)=b$ for all $h \in T$. For any $h \in S \backslash\{i\}$ by fairness we have $\varphi_{h}(v)-$ $\varphi_{h}(\chi)=\varphi_{j}(v)-\varphi_{j}(\chi)$ since then $h$ and $j$ are symmetric players in the game $c_{S} w_{(S \backslash\{i\}) \cup\{j\}}$. Therefore, by $(11)$ we have $\varphi_{h}(v)=\varphi_{j}(v)-\varphi_{j}(\chi)+\varphi_{h}(\chi)=$ 
$b-\left(c_{T} /|T|-c_{S} /|S|\right)$ for $h \in S \backslash\{i\}$. According to Theorem 2(c) we can also conclude that $\varphi_{i}(v)=\varphi_{d}(v)$ for all $d \in S$, since $i$ and any $d$ in $S$ are symmetric. Thus

$$
\varphi_{i}(v)= \begin{cases}b-c_{T} /|T|+c_{S} /|S| & \text { if } i \in S, \\ b & \text { if } i \in T .\end{cases}
$$

But quasi-efficiency implies

$$
c_{S}+c_{T}=\sum_{K \subseteq N} v(K)=\sum_{i=1}^{n} \varphi_{i}(v)=n \cdot b-\frac{|S|}{|T|} c_{T}+c_{S} .
$$

Hence the constant $b$, and thereby the value $\varphi_{i}(v)$ for $i \in N$, are uniquely determined for $n \geq 3$.

Now, let $n=2$. Therefore $N=\{1,2\}$. Then $v=c_{\{1\}} w_{\{1\}}+c_{\{2\}} w_{\{2\}}$. Let $\varphi_{2}(v)=a$. The zero-player property implies that $\varphi_{2}\left(\left(c_{\{1\}}-c_{\{2\}}\right) w_{\{1\}}\right)=0$. By quasi-efficiency we have $\varphi_{1}\left(\left(c_{\{1\}}-c_{\{2\}}\right) w_{\{1\}}\right)=c_{\{1\}}-c_{\{2\}}$. Fairness implies that

$$
\varphi_{1}(v)-\varphi_{1}\left(\left(c_{\{1\}}-c_{\{2\}}\right) w_{\{1\}}\right)=\varphi_{2}(v)-\varphi_{2}\left(\left(c_{\{1\}}-c_{\{2\}}\right) w_{\{1\}}\right)
$$

and so $\varphi_{1}(v)=a+c_{\{1\}}-c_{\{2\}}$. From the quasi-efficiency axiom we obtain $c_{\{1\}}+c_{\{2\}}=\varphi_{1}(v)+\varphi_{2}(v)=2 a+c_{\{1\}}-c_{\{2\}}$, and hence $a=c_{\{2\}}$.

Thus $\varphi(v)$ is uniquely determined and because it satisfies all axioms mentioned in Theorem 7 , by part $(\Rightarrow)$, we finally have $\varphi(v)=\operatorname{DP}(v)$.

2.3. Proof of Theorem 8. $(\Rightarrow)$ It is known that the least square prenucleolus (L) satisfies the efficiency, average marginal contribution monotonicity (AMC), and inessential game and additivity axioms (cf. Theorem 5). It is easy to verify that additivity implies inter-game balance. On the other hand, the LS-prenucleolus is a symmetric value; therefore it also has the equal treatment property. Thus, by Theorem 2(a) it satisfies fairness.

$(\Leftarrow)$ Fix a value $\varphi$ on $G_{N}$ and assume that it satisfies all the conditions mentioned in the theorem. For $S \subseteq N$, let $w_{S} \in G_{N}$ be a basic game defined in (8).

By efficiency and AMC, there exist constants $\alpha_{S}, S \subseteq N$, such that for real $c_{S}$,

$$
\varphi_{i}\left(c_{S} \cdot w_{S}\right)= \begin{cases}c_{S} \cdot \alpha_{S} /|S| & \text { if } i \in S \\ -c_{S} \cdot \alpha_{S} /(n-|S|) & \text { if } i \notin S\end{cases}
$$

with $\alpha_{N}=1$.

Let $S_{1}$ and $S_{2}$ be two disjoint nonempty subsets of $N$. Then, by AMC, we have $\varphi_{i}\left(w_{S_{1}}+w_{S_{2}}\right)=\varphi_{j}\left(w_{S_{1}}+w_{S_{2}}\right)$ for $i \in S_{1}, j \in S_{2}$. Thus, by inter-game balance it follows that $\varphi_{i}\left(w_{S_{1}}\right)-\varphi_{j}\left(w_{S_{2}}\right)=\varphi_{j}\left(w_{S_{1}}\right)-\varphi_{i}\left(w_{S_{2}}\right)$, whence, by $(12)$,

$$
\frac{\alpha_{S_{1}}}{\left|S_{1}\right|}-\frac{\alpha_{S_{2}}}{\left|S_{2}\right|}=-\frac{\alpha_{S_{1}}}{n-\left|S_{1}\right|}+\frac{\alpha_{S_{2}}}{n-\left|S_{2}\right|},
$$


and consequently,

$$
\alpha_{S_{1}} /\left(\left|S_{1}\right|\left(n-\left|S_{1}\right|\right)\right)=\alpha_{S_{2}} /\left(\left|S_{2}\right|\left(n-\left|S_{2}\right|\right)\right)
$$

if $S_{1} \neq \emptyset, S_{2} \neq \emptyset$ and $S_{1} \cap S_{2}=\emptyset$.

We will now prove that (13) holds for all nonempty subsets of $N$. If $S_{1} \cap S_{2} \neq \emptyset$ and $S_{1} \cup S_{2} \neq N$ then (13) is true for $S_{1}$ and $N \backslash\left(S_{1} \cup S_{2}\right)$ as well as for $S_{2}$ and $N \backslash\left(S_{1} \cup S_{2}\right)$, and therefore for $S_{1}$ and $S_{2}$. If $S_{1} \cap S_{2} \neq \emptyset$ and $S_{1} \cup S_{2}=N$ then (13) holds for $S_{1}$ and $N \backslash S_{1}$ as well as for $S_{2}$ and $N \backslash S_{2}$, and thus for $N \backslash S_{1}$ and $N \backslash S_{2}$ and finally for $S_{1}$ and $S_{2}$. Then

$$
\alpha:=\alpha_{S} /(|S|(n-|S|))
$$

for any nonempty $S \subsetneq N$ is a constant. Thus $\alpha$ is well defined when $n=$ $|N|>1$. For $|N|=1$, let $\alpha$ be arbitrary.

Let

$$
\lambda_{i}(v)=\frac{v(N)}{n}+\alpha\left(n \sum_{\substack{K \subseteq N \\ i \in K}} v(K)-\sum_{p \in N} \sum_{\substack{K \subseteq N \\ p \in K}} v(K)\right)
$$

for any $i \in N$.

We will prove that the value $\varphi$ is uniquely determined as $\varphi_{i}(v)=\lambda_{i}(v)$ for any $i \in N$.

We apply all the assumptions and notations concerning the game graph method introduced in Subsection 2.2.

It is clear (by the efficiency axiom and (15)) that $\varphi=\lambda$ for all one-player games. Suppose that $\varphi=\lambda$ for all $m$-person games where $m \leq n-1$, and let $v$ be an $n$-person game. We will use induction on $d(v)$.

If $d(v)=0$, then the efficiency and AMC properties imply that $\varphi_{i}(v)=$ $0=\lambda_{i}(v)$ for any $i \in N$. If $d(v)=1$ then $v=c_{S} w_{S}$ for some $S \subseteq N$. By (12), (14) and (15) we easily get $\varphi_{i}(v)=\lambda_{i}\left(c_{S} \cdot w_{S}\right)$ for all $i \in N$.

Assume therefore that $\varphi(z)$ coincides with $\lambda(z)$ for all games $z \in G_{N}$ with $d(z) \leq k$ (for some $k \geq 1$ ) and let $d(v)=k+1$. Then $d(v) \geq 2$ and $n \geq 2$. We consider two cases.

CASE 1: $N$ is a component in $\Gamma_{v}$. Fix any $j \in \vartheta(v)$ and let $S_{0}=\{j\}$, where $\vartheta(v)$ and $S_{b}$ are defined as in Subsection 2.2. We know that there exists an $m \in N$ such that $S_{0}, S_{1}, \ldots, S_{m}$ is a partition of $N$ consisting of nonempty sets. We can repeat those considerations for $i \in S_{t}$ and some $h \in S_{t-1}$ to get $a_{i}=a_{h}-\lambda_{h}\left(v-c_{S} \cdot w_{S}\right)+\lambda_{i}\left(v-c_{S} \cdot w_{S}\right)$, with some constant $a_{h}$ determined inductively. It follows that all $a_{i}$ with $i \in S_{t}$ are uniquely determined, implying the same for all $a_{i}, i \in N$. Just as in Subsection 2.2 , we can prove that $a$ and, consequently, the values $\varphi_{i}(v), i=1, \ldots, n$, are uniquely determined. Hence $\varphi_{i}(v)=\lambda_{i}(v)$ for all $i \in N$, because $\lambda$ satisfies the first four axioms considered in Theorem 8 (we have not used the inessential game property of $\varphi$ yet). 
CASE 2: $N$ is not a component in $\Gamma_{v}$. In exactly the same way as in Case 2 in Subsections 2.1 and 2.2 we conclude that $\Gamma_{v}$ has exactly two components, denoted by $S$ and $T$, with $S \cap T=\emptyset$ and $S \cup T=N$, and therefore $v$ is the sum of two basic games: $v=c_{S} w_{S}+c_{T} w_{T}$ with $c_{S} \neq 0$ and $c_{T} \neq 0$.

Since $g_{i}\left(c_{S} w_{S}+c_{T} w_{T}\right)=g_{j}\left(c_{S} w_{S}+c_{T} w_{T}\right)$ for $i, j \in S$, the efficiency and $\mathrm{AMC}$ axioms imply that there exists a constant $\beta_{S}$ such that

$$
\varphi_{i}\left(c_{S} w_{S}+c_{T} w_{T}\right)= \begin{cases}\beta_{S} /|S| & \text { if } i \in S, \\ -\beta_{S} /(n-|S|) & \text { if } i \in T\end{cases}
$$

(note that $|T|=n-|S|$ ).

Define an $n$-person game $z=c_{S} w_{S}+c_{T} w_{T}+\left(c_{S}-c_{T}\right) w_{T}=c_{S}\left(w_{S}+w_{T}\right)$. By the AMC axiom we have $\varphi_{i}(z)=\varphi_{j}(z)$ for any $i \in S$ and $j \in T$ and then, according to the inter-game balance property,

$\varphi_{i}\left(c_{S} w_{S}+c_{T} w_{T}\right)-\varphi_{j}\left(c_{S} w_{S}+c_{T} w_{T}\right)=\varphi_{j}\left(\left(c_{S}-c_{T}\right) w_{T}\right)-\varphi_{i}\left(\left(c_{S}-c_{T}\right) w_{T}\right)$.

Hence, using (16) and (12), we get

$$
\frac{\beta_{S}}{|S|}+\frac{\beta_{S}}{n-|S|}=\left(c_{S}-c_{T}\right) \alpha(n-|T|)+\left(c_{S}-c_{T}\right) \alpha|T|=\left(c_{S}-c_{T}\right) \alpha \cdot n
$$

and therefore

$$
\beta_{S}=\frac{\left(c_{S}-c_{T}\right) \alpha \cdot n \cdot|S| \cdot(n-|S|)}{|S|+n-|S|}=\left(c_{S}-c_{T}\right) \alpha|S|(n-|S|) .
$$

Now, using (16), (14) and (15) we deduce that $\varphi_{i}(v)=\lambda_{i}(v)$ for any $i \in N$.

This way we have proved that $\varphi$ coincides with $\lambda$ on $G_{N}$. The inessential game property (applied to $\lambda$ ) implies that $\alpha=1 /\left(n 2^{n-2}\right)$. Thus, we finally obtain $\lambda=\mathrm{L}$. The proof is complete.

2.4. Proof of Theorem 9. $(\Rightarrow$ ) It is well known (cf. L. M. Ruiz et al. (1996)) that the LS-nucleolus (LN) satisfies the super-efficiency, average marginal contribution monotonicity and inessential game axioms. We must prove that it also has the inter-game balance and fairness properties.

One can easily check that for any $v \in G_{N}$ and $i, j \in N, i \neq j$, we have (cf. L. M. Ruiz et al. (1996))

$$
\mathrm{LN}_{i}(v)-\mathrm{LN}_{j}(v)=\frac{1}{2^{n-2}}\left(g_{i}(v)-g_{j}(v)\right) .
$$

Suppose that $v, w \in G_{N}$ and $\operatorname{LN}_{i}(v+w)=\operatorname{LN}_{j}(v+w)$ for some $i, j \in N$, $i \neq j$. Then by (17), we have $g_{i}(v+w)=g_{j}(v+w)$. Thus, by additivity of $g$, we obtain $g_{i}(v)-g_{j}(w)=g_{j}(v)-g_{i}(w)$ and by $(17), \operatorname{LN}_{i}(v)-\operatorname{LN}_{j}(w)=$ $\mathrm{LN}_{j}(v)-\mathrm{LN}_{i}(w)$. Therefore the LS-nucleolus satisfies the inter-game balance axiom.

Let $w \in G_{N}$ and $i, j \in N, i \neq j$, be two players such that $w(S \cup\{i\})=$ $w(S \cup\{j\})$ (i.e. players $i, j$ are symmetric in the game $w)$. Then we have $g_{i}(w)=g_{j}(w)$. Therefore 


$$
g_{i}(v+w)-g_{i}(v)-g_{i}(w)=g_{j}(v+w)-g_{j}(v)-g_{j}(w)
$$

and $g_{i}(v+w)-g_{i}(v)=g_{j}(v+w)-g_{j}(v)$ for any $v \in G_{N}$. By (17) we have $\mathrm{LN}_{i}(v+w)-\mathrm{LN}_{i}(v)=\mathrm{LN}_{j}(v+w)-\mathrm{LN}_{j}(v)$. Thus the LS-nucleolus satisfies fairness.

$(\Leftarrow)$ Suppose that a value $\varphi$ on $G_{N}$ satisfies all axioms given in the theorem. Then it is an imputation satisfying the assumptions of Theorem 8 . According to that theorem and the conclusions $\left({ }^{5}\right)$ made by L. M. Ruiz et al. (1996) we have $\varphi=\mathrm{LN}$.

Acknowledgements. I thank Professor José M. Zarzuelo (University of the Basque Country, Bilbao, Spain) for useful explanations concerning the properties of the LS-nucleolus. I am also grateful to the anonymous referee for patient and careful reading of the paper as well as for detailed comments and suggestions, which were helpful to improve my work.

\section{References}

J. F. Banzhaf III (1965), Weighted voting does not work. A mathematical analysis, Rutgers Law Review 19, 317-343.

R. van den Brink (2001), An axiomatization of the Shapley value using a fairness property, Internat. J. Game Theory 30, 309-319.

J. Deegan and E. W. Packel (1979), A new index of power for simple n-person games, ibid. 7, 113-123.

E. Lehrer (1988), An axiomatization of the Banzhaf value, ibid. 17, 89-99.

A. S. Nowak (1997), On an axiomatization of the Banzhaf value without the additivity axiom, ibid. 26, 137-141.

L. M. Ruiz, F. Valenciano and J. M. Zarzuelo (1996), The least square prenucleolus and the least square nucleolus. Two values for TU games based on the excess vector, ibid. $25,113-134$.

L. S. Shapley (1953), A value for n-person games, in: H. W. Kuhn and A. W. Tucker (eds.), Contribution to the Theory of Games II, Ann. of Math. Stud. 28, Princeton Univ. Press, Princeton, 307-317.

H. P. Young (1985), Monotonic solutions of cooperative games, Internat. J. Game Theory $14,65-72$.

Central Statistical Office

Statistical Office in Poznań

Branch in Kalisz

Pl. J. Kilińskiego 13

62-800 Kalisz, Poland

E-mail: amlodak@stat.gov.pl

Received on 16.7.2002;

revised version on 23.4.2004

$\left(^{5}\right)$ An LS-prenucleolus of a game $v$ which is an imputation of this game coincides with its LS-nucleolus. 